\title{
A Research on Cyber Security Awareness based on Big Data
}

\author{
Sujit, Syed nabeel azeez, Taurunika Shivashankaran, Gururaj H L
}

\begin{abstract}
In digital word cybersecurity is to help us to prevent attacks on network resources, private information and vital certifications of an association. The fundamental objective of this survey paper is to focus on the various types of cyber-attacks and their quick fix to how we can protect our self from such attacks. Other then, it also gives information about the various forms of cyber-crime and its protection worldwide. These days, with wide usage of internet services with low-cost everyone, is using the net. But many of them don't know about cyber fraud i.e.: how attackers steal their data or personal information and sell to others, this paper helps to create awareness of cybersecurity for such people. So as to determine digital security issues, the security specialist's locale including government segment, the scholarly community, the private part should cooperate to comprehend the rising dangers to the processing scene. This paper presents a specialized, social and moral association uninhibitedly sharing digital assault angle in the soul of worldwide co-task bridging geo-political fringes by conveying dynamic checking.
\end{abstract}

Keywords: cyber-crime, cyber-attacks, digital security.

\section{INTRODUCTION}

Advanced security isn't limited to an individual workstation, yet what's more used to cover information of individual PDAs like mobile phones and tablets since these devices have ended up being the essential vehicle of information trade in light of the present movements in development [2]. So as to choose propelled security issues, the security researcher's district including governance territory, the academic network, the private division must coordinate to fathom the rising threats to the figuring scene. This paper displays a particular, social and good affiliation wholeheartedly sharing computerized strike angles in the spirit of overall co-assignment crossing geo-political edges by sending dynamic watching [2]. Even more vitally it exhibits a significantly new and progressively qualified trained crew to recognize future, present and past advanced warnings by improved "colossal data" examination and another strategy Current mechanical assemblies and frameworks wait behind the computerized ambushes and can't be robotized as the aggressors are individuals and can circumvent controls.

Revised Version Manuscript Received on August 19, 2019.

Sujit,Department. of Computer Science and Engineering, Vidyavardhaka College of Engineering, Mysuru, Karnataka, India.

(email:sujithashapur123@gmail.com)

Syed nabeel azeez, Department. of Computer Science and Engineering, Vidyavardhaka College of Engineering, Mysuru, Karnataka, India. Engineering, Vidyavardhaka College of Engineering, Mysuru, Karnataka, India. Vidyavardhaka College of Engineering, Mysuru, Karnataka, India.
Taurunika Shivashankaran, Department. of Computer Science and

Gururaj H L, Department. of Computer Science and Engineering,

The issue is that security endeavors are troublesome and complicated. We fail to focus and don't notice the precedents that we must and less Armed with the learning down to fragment/piece levels and taking on a comparative attitude as a developer, it is absolutely conceivable that a lone individual can possibly make calamitous disillusionment essentially any fundamental system, meanwhile Big Data examination ensures significant open entryways for neutralizing activity and recognizable proof of bleeding edge computerized ambushes using related inside and external security data . Speedy enhancements in development give progressing degree of profitability to affiliations that prompts the colleague of basic perils with the data and information of affiliations. Advanced and better security communicates the affirmation of data, structures, and frameworks in the computerized world which is a tyrannical endeavor for all business affiliations. Advanced security will be astoundingly significant as the number of contraptions related with the web will construct, which will be at a quick speed. Computerized risks can be delegated below [1] they are:

- Cyberterror

- Cybercrime

- Cyberwar

\section{LITERATURE REVIEW}

In [1] the author has focused on the vulnerable "CYBERSPACE OF INDIA" which can be easily exploited. There is an augmentation in the online mechanized hits occasions with the quick improvement of Internet use in recent years. In the year 1975 NI C's in India were created so as to give plans identified with IT to the government. Rule deals with that were set up around then were:

(a) INDONET: - To interface IBM united PC server which made up PC foundation

(b) NIC NET: Bury arranges worn in open relationship to interface Center with the state, and unmistakable relationship at the district level. [6]

(c) ERNET: - ERNET addresses Education Research Network and is utilized to fill the need for scholastics and research frameworks. [6]

[1] Telecommunication, Space, Energy, Defense, Transport and other open associations are crucial zones that basically subject to PC systems to pass on information, for correspondence also like the business reason. In this manner, there is a goliath effect of utilizing the Internet in these fields as wellspring of data and correspondence as shown by NBSA 
confident arrangement to construct the web affiliation which was released by the government of India, correspondence passage and E-displaying yet government should make use of security techniques which are stronger for computerized bad behavior and misleading advanced raids.

In [2] the author cites information about "WEAPONIZATION OF CYBERSPACE".As it is observed the best and basic necessity for all associations is mechanized security and that the web keeps being an always dangerous place for all users. The advancing halfway insistence of weaponization of the web; bring your own least common contraption (BYOD); cloud responsibilities; moved social building, and the bound-to-be completely sent Version 6 of IP can essentially moreover tangle the blend.

Specifically, by and large, trust has been similarly isolated among countries over the Dudu, Stuxnet, Gauss, and Flame between related worms, much like Aurora finished a few years sooner. Released in the wild, these worms will, or as is generally the condition, had started late been understands and its combinations used to strike express targets.

This paper [2] puts forward a fundamental strategy which utilizes "dynamic" create security checking, not in itself another idea, yet cross-associating this with system stream estimations. In this way on the off chance that either approach neglects to satisfactorily perceive an eccentricity, by then the blend with human information will either declare a strike or a phony positive. This guarantees the modernized security present is ideal for the relationship by feasibly learning and dependably thriving checking within the structure. This is beside improved by the dynamic sharing of cutting-edge danger estimations on an overall reason.

In [3] the author explains about THE "HADOOP ECOSYSTEM". Big Data framework for getting ready and examination involves different programming instruments. At present the Hadoop programming condition (Fig. 1) is to be considered as an equivalent word for the "Huge Data". Hadoop acknowledges MapReduce progression of Google, which gives adjusted information paralleling and preparing on PC packs. A significant number of the Hadoop parts are open source programming made in different Apache undertakings. Explicit pieces of Hadoop characteristic structure are given underneath in short portrayal:

- HDFS (Hadoop Distributed File System)

- MapReduce

- Apache Pig

- Hive

- HCatalog

- HBase

- Zookeeper

- Mahout

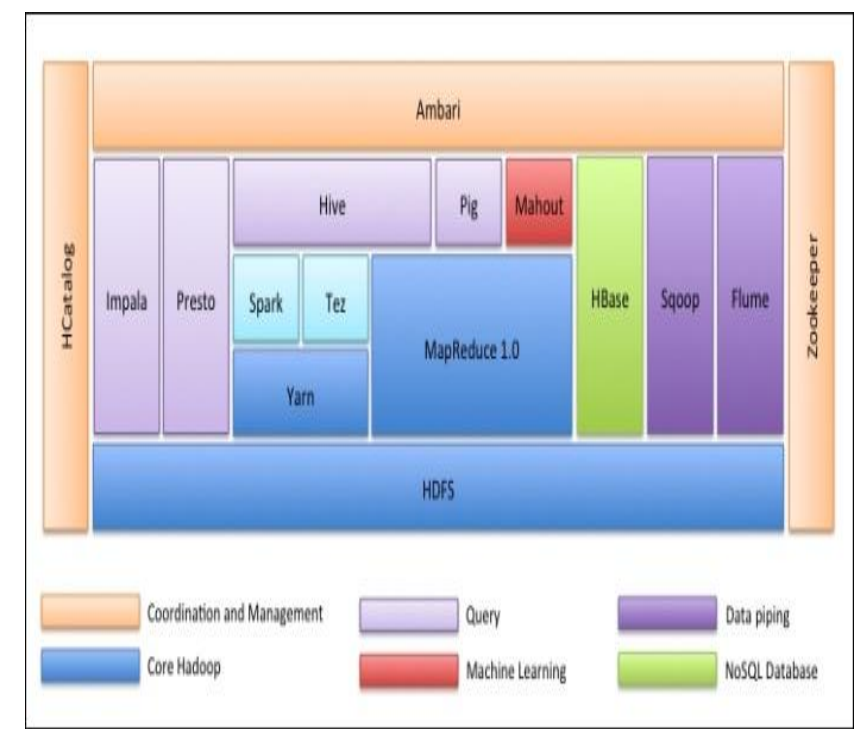

Figure 1: Apache Hadoop Ecosystem [3]

\section{A. HDFS (Hadoop Distributed File System)}

A passed-on record structure for limit and the leading body of date stockrooms from several terabytes to petabytes; it is the focal point of the Hadoop. HDFS parts the data into squares and doles out these squares on servers in better places administered to them. The TCP/IP level is used for correspondence. HDFS is accused tolerantly, and frustration of any part does not impact the general structure execution.

\section{B. MapReduce}

Executes (in Java) Google's scattered handling model for parallel figuring with immense data, a couple of petabytes, in PC gatherings. MapReduce work contains two phases: Map and Reduce. On the Map-step the data is pre-dealt with. To do this, one of the PCs (known as the expert center) gets input data of the issue, separates them into parts and trades to various PCs (master centers) for pre-getting ready. On the Reduce-step the pre-dealt with data is assembled. The pro center point gets responses from the working centers and structures the game plan dependent on their results.

\section{Apache Pig}

Apache Pig fragment involves a compiler that makes a gathering of MapReduce ventures, and language 'Pig Latin'. Offers assistance for performing SQL-like inquiries to dispersed databases to Hadoop.

\section{Hive}

Hive a data circulation focus establishment, used to insinuate significant data set in the Hadoop record system through SQL.

\section{E. HCatalog}

HCatalog Gives amassing the administrator's organization and data tables made in Hadoop. It reinforces supportable working of the Hadoop portions, for instance, Pig, MapReduce, Streaming, and Hive. 


\section{F. HBase (Hadoop Database)}

HBaseS passed on, columnar database (got from Google's Bigtable).

\section{G. Zookeeper}

Zookeeper its guideline work is to store the coordination information, naming, giving scattered synchronization, and social occasion organizations, which are fundamental for a combination of passed on systems.

\section{H. Mahout}

Mahout programming for AI, including key estimations, for instance, gathering, packing, and proposal and synergistic isolating. Crucial figurings are executed with Map/Reduce perspective on the Hadoop upper measurement.

In [4] author has focused on Cybersecurity Insurance and Business Strategies: The motivation driving finishing CI is to constrain the costs to cause by different moved occasions, weave electronic strikes, harmful wear out, overseeing needs, data spillage, and business impedance. It is a required execution in unequivocal undertakings analyzed for after by the business laws or measures. Beginning at now, there are a few CI plot models open for endeavors to lessen or diminish the dangers seen from the above-mentioned reasons.

The first association show is Prevention Promotion Model that bases on imagining dangers by examining progressing or past structures, by which the dangers are possibly troubled. This structure can help clients with diminishing the level of automated strikes since the enduring status checks can see most ordinary uncommon practices. The other model is empowering capricious state security undertaking that enough affirmation information. This system if all else fails requires a gutsy structure resuscitate Both alliance models needs a wide piece of confirmation thought and express sponsorships.

Looking out for this issue, the Department of Homeland Security (DHS) of the United States pushed a strategy working session concentrating on the CI business center. Three proposals were made by CI specialists, including sharing CI data, looking edge strikes' results, and empowering cybersecurity chance the experts. Likewise, a proposed outline was considered, which utilized a Cyber Incident Data Repository (CIDR) to help information sharing, modernized occasion evaluations, and risk examinations.

Regardless, a strong procedure is beginning in the not so far away past not got yet before gets some data about had separated this field. The cost of CI is so far at a hard to miss state with an inducing center around that captivating business shapes are bound by the CI-related models. Distinctive business divisions are not dynamic in utilizing IT-based configurations in light of the shocking financial trouble accomplished by CI. This reasonable peculiarity does not deal with the essential motivation driving stirring up CI, which expects to ensure relationship by including security respects.

\section{SECURITY THREATS AND SECURITY\& RESULTS}

\section{A. Assault on IOT:}

Operators are constantly understanding the progress of the Internet of Things headway and how it is energizing to push information. In any case, incredibly, advanced subject get-togethers are in search for after of them. As showed up by IDC, forty-six component of the IT chief offered a light to encounter security scenes related to strikes on the IoT devices. It was revealed in the examination that around ninety-three parts of the respondents got upon out throwing the experts in IoT security to manage the discussion. Subsequently, it was found by seventy-one percent of respondents that IoT dangers are more over the top to pick than the standard security events.

The nonappearance of sensible consistency makes sagacious contraptions unprotected against the most basic maltreatment of security. On snatching guileful endorsement get to, programming specialists can bargain Internet of Things structures in different ways, with DDoS strikes being the best concern. Envision countless coolers, toothbrushes, and vehicles inciting maintained strikes on corporate structures. It's an astonishing idea when joined with the present accumulate of contraptions usually utilized in DDoS assaults. Focusing on unpredictable IoT information through ransomware is another probability that has security aces on caution.

\section{B. Ransomware attack-as-a-Service:}

While discussing ransomware, security analyzers beware of the remote possibility that it will keep on being a fundamental hazard in 2018. In their Annual Cybersecurity Report documentation, Cisco assesses that these impulse grounded ambushes will be made at a yearly check of three hundred-fifty rates. Additionally, that improvement has gone to the square of unfathomable giving dazzling setbacks. The programming security firm, bit protect evaluates that ransomware is correct now a \$2billion as a rule adventure.

Ransomware - as - a - service (RaaS) recognized a tremendous development in the progress of ransomware. Beginning from the most essential terminations of the diminish web, RaaS outfits juvenile programming engineers with devices that make it possible to execute impossibly refined and compensating ambushes. These stages work an extraordinary game-plan like astounding programming scattering affiliations, offering full-get to licenses, month to month in regards to plans, and even dedicated explicit help. With RaaS determinedly progressing past any binding impact web, one can foresee that the model ought to furthermore make in the midst of 2018.

\section{Cloud-based Malware:}

The more essential the Cloud makes; dynamically detectable target it moves toward persuading the chance to be aggressors. While genuine Cloud dealers address an increasingly noticeable score for modernized guilty parties, the unimportant perceived affiliations may be considerably progressively vulnerable. 
Minimal expert concentrates, for the most part, miss the mark on the establishment and resources which the business control houses, like Amazon, Google, and Microsoft, use to hinder security risks. The vulnerabilities would in like way making these increasingly humble providers bound to surrender the ransomware demands. This is the finish of MIT Technologies ponder, which anticipate he constantly fundamental the cloud develops, the more noticeable target it pushes toward observing the chance to be for aggressors. While the authentic cloud operators address an undeniably vital help for cutting edge punks, the unimportant saw affiliations can be persistently unprotected. Constantly minimal expert focuses typically come up short on the structure and assets which industry powerhouses, similar to Amazon, Google, and Microsoft, use to impede security dangers. These vulnerabilities could in like way make those humbler suppliers bound to surrender to ransomware requests. That is the completion of the MIT Technology Review, which predicts that ransomware will go for the cloud in 2018.ts that ransomware will go for the cloud in 2018.

\section{AI Threats:}

Man-made mental bent has been idolized for its capacity to inquire about information, see talk designs, and perform assorted assignments that normally require human data.

Anything is possible, in any case, that new potential applies to unsafe use additionally. Outfitted with AI, designers could execute surprising state strikes that cripple corporate systems similarly as change vehicles and robots into dangerous weapons. As indicated by a Webroot consider, 91 percent of IT security masters passed on worries over AI being utilized to strike affiliations.

\section{E. Insider Attacks:}

Most request plan is depended on to execute perils emanating from the outside of the affiliation. Notwithstanding, get a couple of data about the support that there should be a reliably clear redesign on insider perils. Data security uncovered that inside scenes were being responsible for forty-three component of the data breaks 0.5 cognizant, half fortuitous. The other report found that seventy-four component of the affiliations trust that they are unprotected against inward strikes. An in each pragmatic sense unclear report embraced that these inside strikes are among the costliest to pick, with fifty-three percent of respondents looking at costs more them $\$ 100,000$.

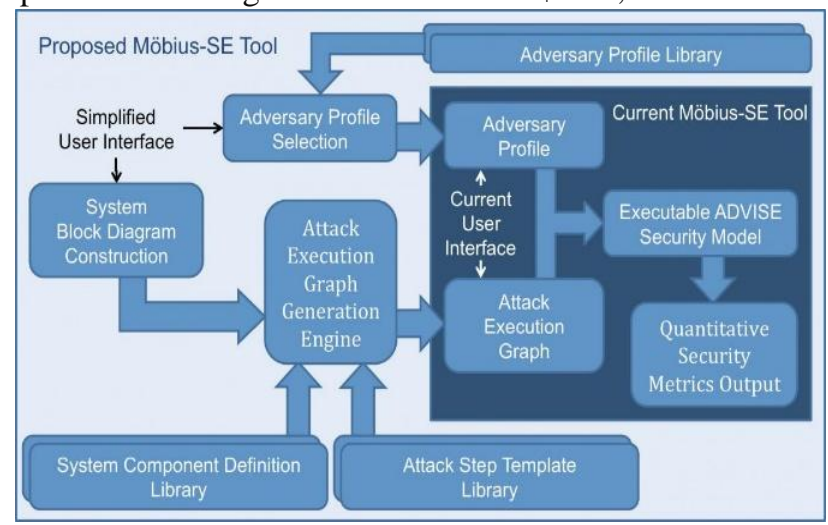

Figure 2: security tool to help predict, prevent cyber-attacks

\section{F. steps to prevent cybercrime and attacks:}

Make a safe pushed regular framework inside the country, produce enough trust similarly as trust in the IT business and trades the web along these lines improve get-together of IT in each piece of the overall population. Engage convincing evasion, examination, and arraignment of cybercrime and update of low underwriting limits through fitting conclusive mediation. By conceptualizing new programming improvement sorts of advancement and practices of structure arranging essential fragments can be guaranteed. Unfathomable data and getting ready should be given to IT security to support them. Some convincing data and information exchange and fast specific evidence ways should be backtracked up to stop these undermining events in the automated universe.

\section{CONCLUSION}

The developers are picked pack. The relationship at each estimation should be in like way enduring, adaptable, and relentless in obliging their fittingness. Having most of the risks in 2018, IT security specialists need to get a handle on a custom dependent on want, fast disclosure, and the speedy answer is the way forward. Make a confirmed pushed regular framework inside the country, produce satisfying desire and trust in IT structure and trades the web in this manner improve task of IT in each piece of the overall population engage Protection of data while in system, overseeing, purpose of imprisonment and travel to watch security of occupant's data and decreasing cash related fiascoes in perspective on electronic terrible direct or data theft.

\section{ACKNOWLEDGEMENTS}

The manuscript is prepared by taking assistance from Accendere Knowledge Management Services Pvt. Ltd, we are thankful to them. We also express our gratitude to our teachers and mentors for guiding us throughout the work.

\section{REFERENCES}

1. S. R. Kumar, S. A. Yadav, S. Sharma, and A. Singh, "Recommendations for effective cyber security execution," in 2016 International Conference on Innovation and Challenges in Cyber Security (ICICCS-INBUSH), 2016, pp. 342-346.

2. V. Patel, "A practical solution to improve cyber security on a global scale," in 2012 Third Worldwide Cybersecurity Summit (WCS), 2012, pp. 1-5.

3. R. Alguliyev and Y. Imamverdiyev, "Big Data: Big Promises for Information Security," in 2014 IEEE 8th International Conference on Application of Information and Communication Technologies (AICT), 2014, pp. 1-4.

4. K. Gai, M. Qiu, and S. A. Elnagdy, "A Novel Secure Big Data Cyber Incident Analytics Framework for Cloud-Based Cybersecurity Insurance," in 2016 IEEE 2nd International Conference on Big Data Security on Cloud (BigDataSecurity), IEEE International Conference on High Performance and Smart Computing (HPSC), and IEEE International Conference on Intelligent Data and Security (IDS), 2016, pp. 171-176. 
5. Z.-W. Lu, "Research about New Media Security Technology Base on Big Data Era," in 2016 IEEE 14th Intl Conf on Dependable, Autonomic and Secure Computing, 14th Intl Conf on Pervasive Intelligence and Computing, 2nd Intl Conf on Big Data Intelligence and Computing and Cyber Science and Technology Congress(DASC/PiCom/DataCom/CyberSciTech), 2016, pp. 933-936.

6. D. Barnes, "Deworming the Internet," Tex. Law Rev., vol. 83, no. 1, p. 51, 2004.

7. N. R. C. and N. A. of Engineering, Toward a Safer and More Secure Cyberspace. Washington, D.C.: National Academies Press, 2007.

8. Z. Yang and J. C. S. Lui, "Security Adoption in Heterogeneous Networks: the Influence of Cyber-Insurance Market," Springer, Berlin, Heidelberg, 2012, pp. 172-183.

9. "The Second Worldwide Cybersecurity Summit EastWest Institute." [Online]. Available: https://www.eastwest.ngo/events/second-worldwide-cybe rsecurity-summit. [Accessed: 05-Feb-2019].

10. M. Glenny, DarkMarket: how hackers became the new media. Vintage, 2012.

11. "National CYber Security Policy," DEiTY. [Online]. Available:

http://deity.gov.in/sites/upload_files/dit/files/Nationa 1 Cyber Security Policy \%281\%29.pdf 